\title{
Patología oral asociada a la sequedad bucal
}

\section{Oral pathology associated with dry mouth}

\author{
Blanco Carrión A*, Otero Rey E**
}

\section{RESUMEN}

El síndrome de boca seca es un importante problema de salud ya que ocasiona intensas alteraciones funcionales y lesiones orales de carácter orgánico. Las alteraciones funcionales son las primeras en aparecer. Existe una dificultad en la masticación, deglución, fonación y alteración en la captación del gusto. Estos problemas pueden desencadenar cambios en la alimentación e incluso comprometer el estado nutricional.

Las lesiones orales de carácter orgánico provocan una alteración de la mucosa oral, aparece brillante, seca, eritematosa, sensible, friable y, en ocasiones, dolorida, facilita la aparición de caries, de evolución rápida y de localización preferentemente cervical; enfermedad periodontal; malestar con el uso de prótesis; predisposición a las infecciones, sobre todo candidiasis; halitosis e incluso manifestaciones extraorales.

Palabras clave: Patología oral, boca seca, caries.

\section{SUMMARY}

Dry mouth syndrome is a major health problem that causes intense functional abnormalities and oral lesions of organic nature. Functional alterations are the first to appear. There is a difficulty in chewing, swallowing, speech and impaired uptake of taste. These problem scan trigger changes in diet and even compromise nutritional status.

Organic oral lesions cause analtered oral mucosa appears bright, dry, erythematous, tender, sore and sometimes friable, facilitates the development of caries, rapidly evolving and preferably cervical location, periodontal disease, discomfort with the prosthetic use, predisposition to infections, especially candidiasis, halitosis and even extraoral manifestations.

Key words: Oral pathology, dry mouth, caries.

Fecha de recepción: 15 de enero de 2014.

Aceptado para publicación: 19 de marzo de 2014.

* PhD, MD, DDS. Máster Odontología Práctica Diaria. Facultad de Odontología. Universidad de Santiago de Compostela.

** PhD, DDS. Máster Odontología Práctica Diaria. Facultad de Odontología. Universidad de Santiago de Compostela.

Blanco Carrión A, Otero Rey E. Patología oral asociada a la sequedad bucal. Av. Odontoestomatol 2014; 30 (3): 129-133.

\section{INTRODUCCIÓN}

El síndrome de boca seca puede suponer un importante problema de salud ya que ocasiona intensas alteraciones funcionales y lesiones orales de carácter orgánico (1). Desde el punto de vista clínico si la disminución de saliva es progresiva y duradera en el tiempo se asociará a signos y síntomas bucales (tanto de origen funcional como orgánico), así como en otras partes del cuerpo. Estos últimos serán de menor o mayor consideración dependiendo de la causa de la hipofunción salival. En un principio, aparecerá una sensación de boca seca (xerostomía) con percepción de sabor amargo y las mucosas estarán más 
sensibles, dolorosas y eritematosas (2). Esta sensación de sequedad sabemos que está relacionada no solo con la cantidad sino también con la calidad de la saliva, de ahí que al afectarse las glándulas salivales menores (secreción muy viscosa y rica en mucina) sea más patente este problema. La clínica puede parecer en un paciente con reserva aparente de saliva.

\section{MANIFESTACIONES ORALES FUINCIONALES}

Son numerosas y progresivas. Son las primeras en aparecer. Existe una dificultad en la masticación, deglución, fonación y alteración en la captación del gusto. No se produce una lubricación de la mucosa oral. La xerostomía nocturna es muy común ya que debido a los ritmos circadianos la saliva alcanza los niveles más bajos durante el sueño (3). El problema se agrava si el paciente es un respirador bucal. El sabor de la comida puede alterarse, la saliva es necesaria para la correcta estimulación de los receptores gustativos. Los pacientes con xerostomía secundaria a radioterapia de cabeza y cuello, a síndrome de Sjögren e, incluso, otras etiologías pueden tener una dificultad añadida para reconocer determinados estímulos gustativos (4). La saliva también es necesaria para preparar los alimentos para la digestión y deglución. Los pacientes con flujo de saliva bajo pueden tener dificultades para masticar y tragar, especialmente los alimentos secos. Estos problemas pueden desencadenar cambios en la alimentación e incluso comprometer el estado nutricional.

\section{MANIFESTACIONES ORGÁNICAS}

\section{Afectación de la mucosa oral}

Pueden verse zonas de atrofia, fisuras y diferentes grados de ulceración en los tejidos blandos, todo ello debido a la falta de lubricación y efecto protector de la saliva. La mucosa aparece brillante, seca, eritematosa, sensible, friable y en ocasiones dolorida. La lengua aparece atrófica con depapilación (Figura 1). El espejo puede quedarse adherido y los labios presentan un aspecto reseco, con descamación y fisuras $(1,5,6)$. Es frecuente una queilitis comisural. La

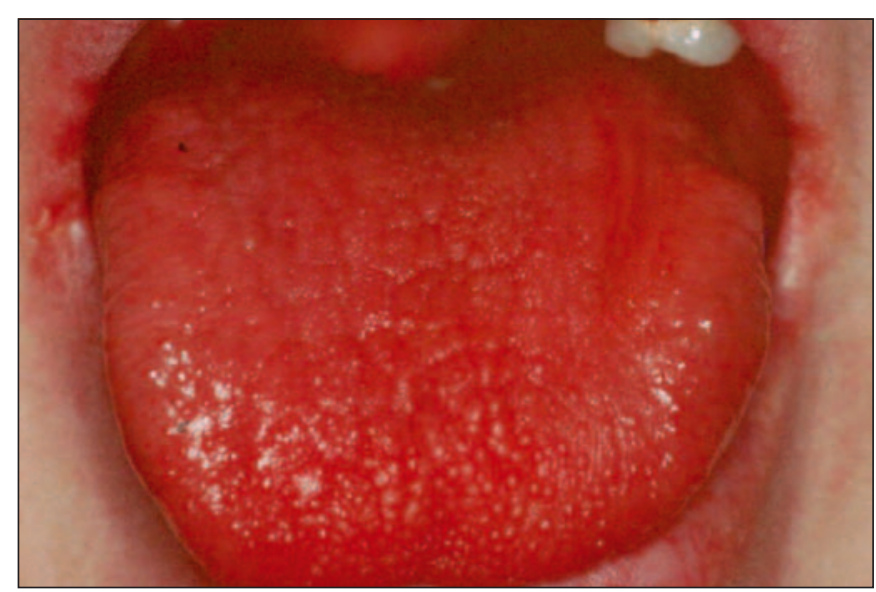

Fig. 1. Mujer con xerostomía e hiposialia. Lengua atrófica depapilada. Fisuración en labios y queilitis comisural.

mucosa se ve especialmente afectada cuando el motivo de la sequedad es por radioterapia (7) o quimioterapia (8).

\section{Caries}

Las alteraciones cualitativas y cuantitativas de la saliva ocasionan un incremento de la caries dental. Son de evolución rápida y de localización preferentemente cervical, incluso en dientes recientemente obturados (Figura 2). También se presentan en lugares atípicos (zonas interproximales e incisales de dientes anteriores). Esta situación es particularmente

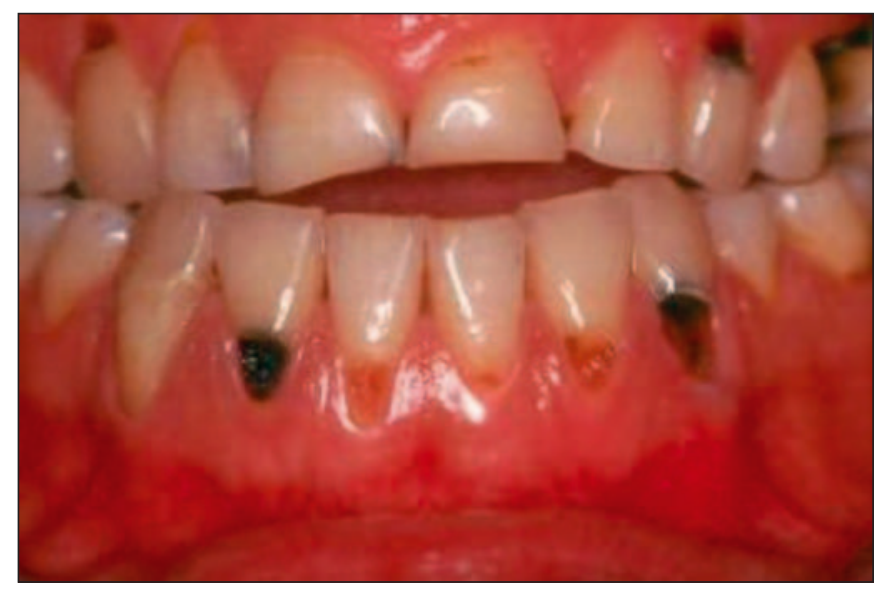

Fig. 2. Caries muy agresivas y múltiples de afectación cervical y radicular. 
patente en personas mayores (muchos de los cuales tienen, en la actualidad, los dientes conservados y con restauraciones en las zonas gingivales) y pueden incluso ocasionar la amputación del diente a nivel coronal (9). La ausencia del efecto tampón de la saliva con respecto al $\mathrm{pH}$, no ayuda al problema permitiendo la colonización por gérmenes especialmente cariogénicos (5,10-13). El problema se agrava de forma especial en los pacientes irradiados (14-17).

\section{Enfermedad periodontal}

La sequedad favorece el acúmulo de placa bacteriana incrementando la severidad y frecuencia de las enfermedades que afectan a la encía. La propia acción limpiadora de la saliva se ve disminuida en unos tejidos periodontales resecos, favoreciendo la inflamación y desarrollando un cuadro de halitosis (favorecido además por las caries) $(1,10,18,19)$. Todo ello puede relacionarse son una mayor facilidad para hacer neumonías, debido a la aspiración de los gérmenes acantonados en las bolsas periodontales (20). El ardor y el malestar bucal en general pueden estar asociados a este problema.

\section{Malestar con el uso de prótesis}

La falta de saliva y lubricación en la interfaz prótesis-mucosa puede producir llagas por roce. La retención de la prótesis puede resultar insuficiente debido a la ausencia de la película salival o la inadecuada viscosidad de la misma (21); aspecto que puede repercutir incluso en un trastorno en la alimentación, especialmente en pacientes ancianos (12).

\section{Predisposición a las infecciones}

Independientemente del posible incremento, ya comentado, de aspiración de gérmenes acantonados en las bolsas periodontales, la falta de saliva provoca una disminución del $\mathrm{pH}$ bucal, ocasiona una disminución de sustancias antiinfecciosas (inmunoglobulinas y lisozima entre otras enzimas) y no existe un sistema de arrastre que aparece fisiológicamente

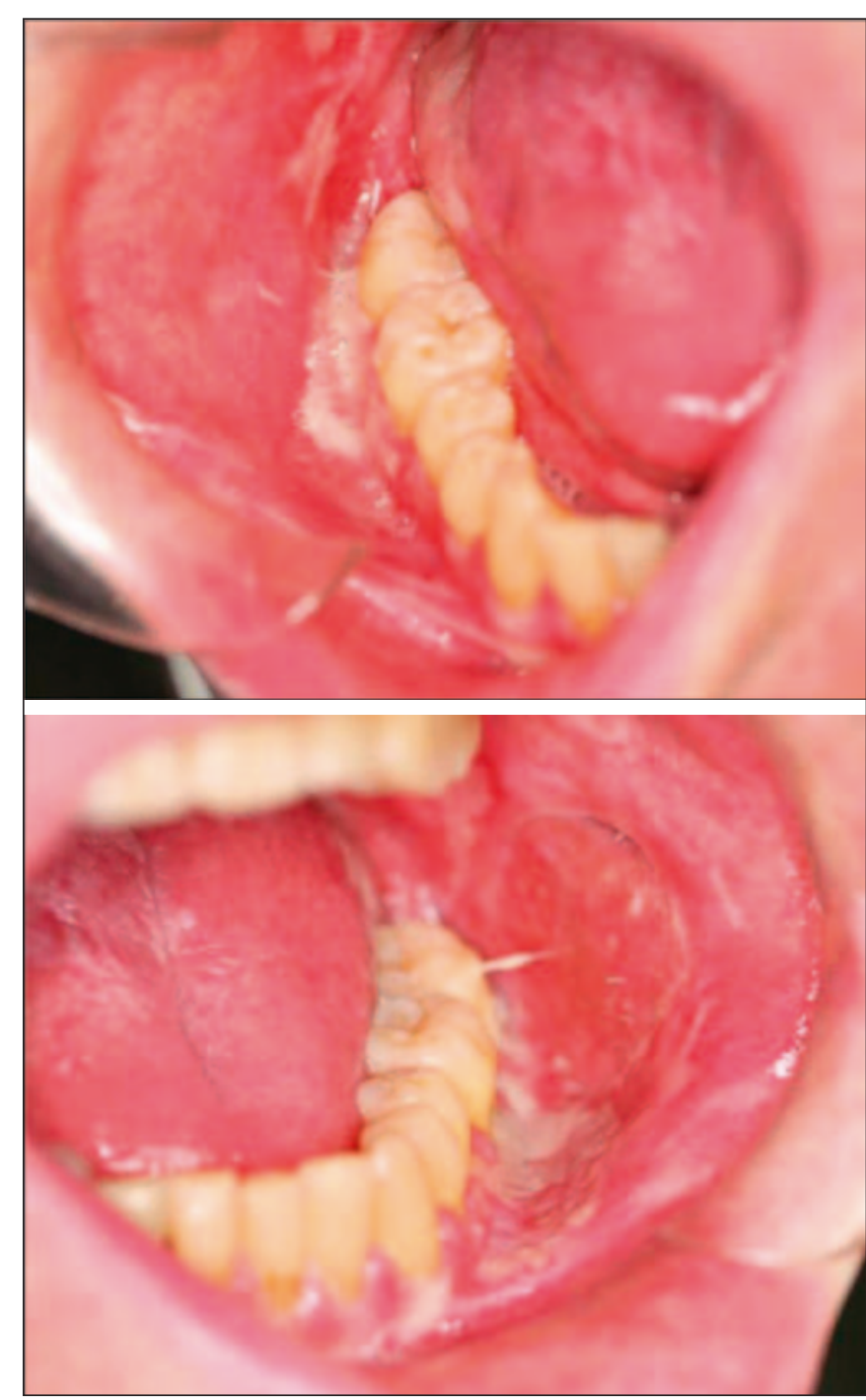

Fig. 3. Candidiasis oral en un paciente sometido a radioterapia. Hiposialia marcada con signos de mucositis.

cuando hay saliva, facilitando todo ello el desarrollo de halitosis e infecciones, de forma muy especial la candidiasis (Figura 3), tanto en el paladar, en la lengua como en los labios en forma de queilitis angular (2). Se pueden presentar en cualquiera de sus formas, siendo la más frecuente, a no ser que haya otros elementos asociados, la forma eritematosa, asociada o no al uso de prótesis $(5,22,23)$. En algunos pacientes, especialmente operados o con síndrome de Sjögren, puede producirse infección de la glándula parótida facilitada por la obstrucción a nivel del conducto de drenaje que ocasiona la hipofunción salival (5). 


\section{Halitosis}

El origen de la halitosis en el $80-90 \%$ de los casos es de origen bucal. La más estudiada es la de origen periodontal, pero la causa principal es la boca seca que facilita el crecimiento bacteriano responsable de la emisión de gases que provoca el mal aliento. La localización más frecuente de estas bacterias es el dorso de la lengua $(18,19)$.

\section{Manifestaciones extraorales}

Además de la sequedad bucal puede aparecer sequedad en la piel con descamación y fisuración, sequedad en otras mucosas como genitales (con prurito, ardor y vaginitis recurrente), en los ojos (con visión borrosa, arenilla y necesidad de lágrimas artificiales), o incluso sequedad nasal y faríngea. No son infrecuentes los problemas reumatológicos sobre todo artritis reumatoidea $(1,5)$.

Todo lo anteriormente comentado hace que los pacientes, en muchas ocasiones tengan intolerancia a comer ciertos alimentos (duros, ácidos, picantes, etc.), produciéndose un círculo vicioso en el que interviene el ardor bucal, la fragilidad de la mucosa (lengua, paladar, mucosa yugal, suelo de boca, orofaringe), la dificultad para hablar y comunicarse en los actos sociales. De esta manera se configura un cuadro de baja autoestima y depresión subclínica desencadenando una retroalimentación del problema de difícil manejo $(24,25)$.

\section{BIBLIOGRAFÍA}

1. López-Jornet P. Alteraciones de las glándulas salivales. Murcia: Universidad de Murcia 2002 p.17-25.

2. Bagán Sebastián JV. Medicina Bucal. Valencia: Medicina oral SL. 2008; 303:278-9.

3. Dias Fernandes CS, Salum FG, Bandeira D, Pawlowski J, Luz C, Cherubini K. Salivary dehydroepiandrosterone (DHEA) levels in patients with the complaint of burning mouth: a casecontrol study. Oral Surg Oral Med Oral Pathol Oral Radiol Endod 2009;108:537-43.
4. Just T, Pau HW, Bombor I, Guthoff RF, Fietkau R, Hummel T. Confocal microscopy of the peripheral gustatory system: comparison between healthy subjects and patients suffering from taste disorders during radiochemotherapy. Laryngoscope 2005; 115:2178-82.

5. Turner MD, Ship JA. Dry mouth and its effects on the oral health of elderly people. J Am Dent Assoc 2007;15S-20S.

6. Rabiei M, Kasemnezhad E, Masoudi rad H, Shakiba M, Pourkay H. Prevalence of oral and dental disorders in institutionalised elderly people in Rasht, Iran. Gerodontology 2010 Sep;3:174-7.

7. Lalla RV, Sonis ST, Peterson DE. Management of oral mucositis in patients who have cancer. Dent Clin North Am 2008;52:61-77.

8. López J, Sabater MM, Muñoz J, Rosello X, Granena A. Evaluation and prevention of oral complications in patients subjected to bone marrow transplantation. A clinical study. Med Oral 2000;5:345-35.

9. Velasco E, Machuca E, Martínez A, Ríos V, Bullón P. Xerostomía en el paciente geriátrico. Arch Odontoest 1994;10:546-53.

10. Silvestre-Donat FJ, Miralles-Jordá L, MartínezMihi V. Tratamiento de la boca seca: puesta al día. Med Oral 2004;9:273-9.

11. Almståhl A, Wikström M. Oral microflora in subjects with reduced salivary secretion. J Dent Res 1999; 78:1410-6.

12. Närhi TO. Prevalence of subjective feelings of dry mouth in the elderly. J Dent Res 1994;73:20-5.

13. Loesche WJ, Bromberg J, Terpenning MS, Bretz WA, Dominguez BL, Grossman NS, Langmore SE. Xerostomia, xerogenic medications and food avoidances in selected geriatric groups. J Am Geriatr Soc 1995;43:401-7.

14. Husein AB, Butterworth CJ, Ranka MS, Kwasnicki A, Rogers SN. A survey of general dental 
practitioners in the North West of England concerning the dental care of patients following head and neck radiotherapy. Prim Dent Care 2011;18:59-65.

15. Sun HB, Gao XJ, Deng J, Li NY, Lu HJ. Progress of oral sequelae during head-neck radiotherapy. Chin J Dent Res 2010;13:51-5.

16. Escoda-Francolí J, Rodríguez-Rodríguez A, Pérez-García S, Gargallo-Albiol J, Gay-Escoda C. Dental implications in oral cancer patients. Med Oral Patol Oral Cir Bucal. 2011;16:e50813.

17. Haveman C, Huber M. Xerostomia management in the head and neck radiation patient. Tex Dent J 2010;127:487-504.

18. Cortelli JR, Barbosa MD, Westphal MA. Halitosis: a review of associated factors and therapeutic approach. Braz Oral Res 2008;1:44-54.

19. Pratibha PK, Bhat KM, Bhat GS. Oral malodor: a review of the literature. J Dent Hyg 2006; 80:8.

20. Loesche WJ, Abrams J, Terpenning MS, Bretz WA, Dominguez BL, Grossman NS, Hildebrandt $\mathrm{GH}$, Langmore SE, Lopatin DE. Dental findings in geriatric populations with diverse medical backgrounds. Oral Surg Oral Med Oral Pathol Oral Radiol Endod 1995;80:43-54.
21. Strahl RC, Welsh S, Streckfus CF. Salivary flow rates: a diagnostic aid in treatment planning geriatric patients. Clin Prev Dent 1990;12:10-2.

22. López J, Jané E, Chimenos E, Roselló X. Actualización de la candidiasis oral. Arch Odont 1997; 13:259-71.

23. Ayuso-Montero R, Torrent-Collado J. López-López J. Estomatitis protésica: Puesta al día. RCOE 2004;9:657-62.

24. Atkinson JC, Wu AJ. Salivary gland dysfunction: causes, symptoms, treatment. J Am Dent Assoc 1994;125:409-16.

25. Rodríguez de Rivera-Campillo E, López-López J, Chimenos-Küstner E. Response to topical clonazepam in patinets with burning mouth syndrome: A clinical stud. Bull Group Int Rech Sci Stomatol Odontol 2010;49:19-29.

\section{CORRESPONDENCIA}

Dr. Andrés Blanco Carrión

Unidad de Medicina Oral

Facultad de Medicina y Odontología.

Universidad de Santiago de Compostela

San Francisco, s/n

15704 Santiago de Compostela, A Coruña

Correo electrónico: andres.blanco@usc.es 